\title{
Las cooperativas de enseñanza en
}

España: Un modelo educativo para el desarrollo profesional docente basado en la economía social

Teaching cooperatives in Spain: An educational model for the professional development of teachers based in the social economy

As cooperativas de ensino na Espanha: um modelo educativo para o desenvolvimento profissional docente baseado na economia social

Recibido. 23 de julio de 2019

Aceptado: 2 de abril de 2020

Publicado: 4 de mayo de 2020

\section{Jesús López Belmonte ${ }^{1}$ Antonio José Moreno Guerrero ${ }^{2}$ Arturo Fuentes Cabrera ${ }^{3}$}

Cómo citar este artículo: López, Belmonte, J., Moreno Guerrero, A. J. y Fuentes Cabrera, A.. (2020). Las cooperativas de enseñanza en España: Un modelo educativo para el desarrollo profesional docente basado en la economía social. Cooperativismo \& Desarrollo, 28(2),1-25. doi: https://doi.org/10.16925/2382-4220.2020.02.01

Artículo de investigación. https://doi.org/10.16925/2382-4220.2020.02.01

1 Universidad Internacional de Valencia -España

Correo electrónico: jesuslopezbelmonte@gmail.com

2 Universidad de Granada, España

Correo electrónico: ajmoreno@ugr.es;

3

Universidad de Granada-España

Correo electrónico: arturofuentes@ugr.es 


\title{
Resumen
}

Las cooperativas de enseñanza son entidades de naturaleza educativa que se encuadran dentro de la economía social. Estas organizaciones aglutinan a un conjunto de personas, unidas por el deseo de satisfacer sus necesidades, tanto laborales como profesionales, para poder desarrollar su vocación docente. En este estudio se ha llevado a cabo una revisión bibliográfica con el fin de aumentar y reforzar la literatura existente acerca del movimiento cooperativo en su espectro educativo, analizado desde un método cualitativo, centrado en un estudio descriptivo y comparado de las diversas fuentes de información, con el propósito de conocer el desarrollo del cooperativismo educativo en España. Debido a su crecimiento en los últimos años, han sido consideradas como una alternativa de centro educativo frente al clasicismo del sistema, conformado por centros públicos y privados. Entre sus metas como entidad pretenden gestionar de manera empresarial un centro educativo, cuyos cimientos se sustentan en la creación y mantenimiento de los puestos de trabajo, para conseguir una estabilidad y tranquilidad laboral entre los profesionales que las integran. Se concluye que estas entidades sociales se han consolidado como una salida profesional de muchos docentes y egresados universitarios.

Palabras clave: economía social, empresa cooperativa, cooperativas enseñanza, tipología; antecedentes; emprendimiento social.

\begin{abstract}
:
Teaching cooperatives are entities of an educational nature that fall within the social economy. These organizations bring together a group of people, united by the desire to meet their needs, both work and professional, to develop their teaching vocation. In this study a literature review has been carried out in order to augment and reinforce the existing literature regarding the cooperative movement in its educational spectrum, analyzed from a qualitative method, focused on a descriptive and comparative study of the various sources of information, with the purpose of understanding the development of educational cooperativism in Spain. Due to their growth in recent years, they have been considered as an alternative form of educational center, when faced with the classicism of the system, made up of public and private centers. Among its goals as an entity, they intend to manage an educational center in an entrepreneurial way, with foundations based on the creation and maintenance of jobs, so as to achieve job stability and tranquility among the professionals involved. It is concluded that these social entities have established themselves as a professional outlet for many university professors and graduates.
\end{abstract}

Keywords: social economy, cooperative enterprise, teaching cooperatives, typology, antecedents, social entrepreneurship.

\section{Resumo:}

As cooperativas de ensino são entidades de natureza educativa que estão inseridas na economia social. Essas organizações reúnem um conjunto de pessoas, unidas pelo desejo de satisfazer suas necessidades, tanto trabalhistas quanto profissionais, para poderem desenvolver sua vocação docente. Neste estudo, é realizada uma revisão bibliográfica com o objetivo de aumentar e reforçar a literatura existente sobre o movimento cooperativo em seu contexto educativo, analisado sob o método qualitativo, centralizado em um estudo descritivo e comparado das diversas fontes de informação, com o objetivo de conhecer o desenvolvimento do cooperativismo educativo na Espanha. Devido a seu crescimento nos últimos anos, tem sido considerado uma alternativa de centro educativo diante do classicismo do sistema, conformado por centros públicos e privados. Uma das metas dessas cooperativas é gerir, de maneira empresarial, um centro educativo, fundamentado na criação e manutenção dos postos de trabalho, para conseguir uma estabilidade e tranquilidade profissional 
entre seus integrantes. Conclui-se que essas entidades sociais vêm se consolidando como uma saída profissional de muitos docentes e graduandos.

Palavras-chave: economia social; empresa cooperativa; cooperativas ensino; tipologia; antecedentes; empreendimento social

\section{Introducción}

Las cooperativas de enseñanza agrupan a un conjunto de personas, ciudadanos de un lugar, que son profesionales de la educación, quienes, por diversas circunstancias, no han podido desempeñar su profesión docente o se ha visto afectada por el cierre de centros, de naturaleza privada, como consecuencia de la crisis económica. Estos profesionales, ante la complicada situación, unidos por las mismas necesidades, aglutinados bajo la misma esperanza de obtener o mantener su puesto laboral, confían plenamente en la fórmula de emprendimiento cooperativo, para mejorar sus vidas y poder desarrollar con total libertad y plenitud su vocación docente.

Tanto ha sido su desarrollo en España, que ya son consideradas como una alternativa, además de una salida profesional para inversores docentes, dentro del sistema educativo, enmarcada entre la escuela pública y la privada; caracterizada por la difusión de los valores intrínsecos de este tipo de entidades y lleva por bandera la calidad e innovación en el servicio educativo que prestan. Legalmente, en su texto jurídico supremo, se presentan diferentes tipos de cooperativas que se pueden implantar en el sector de la enseñanza, en función de su objeto y sus integrantes.

Estas entidades educativas van naciendo con el propósito de dar empleo a las miles de personas, profesionales de la educación, que se encuentran en paro actualmente, afectados por la crisis económica que está atravesando España. Por lo cual muchos optan por seguir formándose, para ofrecer un currículum completo y atractivo para los centros de naturaleza concertada o privada, o bien emigrar a otros países con necesidades educativas, o iniciar el estudio de otra carrera universitaria.

Según los estudios realizados por Fernández (2000), las cooperativas de enseñanza de trabajo asociado son las que producen mayor índice de empleo del conjunto de sociedades cooperativas.

Las cooperativas de enseñanza han originado un aumento de la oferta educativa tradicional. Antiguamente solo se podía elegir entre una enseñanza de carácter pública o privada, en el presente es posible disfrutar de una enseñanza concertada, gracias a este tipo de sociedades que ofrece un matiz diferente, basado en la cooperación, colaboración, compromiso y voluntad de los docentes que a su vez son socios de 
la cooperativa, perseguidores de la eficacia y calidad de la producción, haciendo referencia al proceso de enseñanza-aprendizaje.

Contribuyen a consolidar en la sociedad la fórmula cooperativa y a la expansión y crecimiento del movimiento cooperativo, muchas de las grandes cooperativas españolas de educación sirven de espejo y reflejo para profesionales que están atravesando una difícil situación. Dan lugar a semillas que son regadas por los valores propios que las caracterizan y que culminan en el florecimiento de nuevas cooperativas.

Este modelo de enseñanza se ha visto afectado por la promulgación de las diferentes leyes educativas acontecidas en España, a lo largo de la historia. Estos centros, como cualquier otro público o privado, tienen que adaptar sus recursos, equipamientos e instalaciones en base a las premisas de la ley educativa vigente, para poder cumplir con los requisitos legales y estar a la vanguardia de los mejores centros educativos a nivel nacional, puesto que su futuro va a depender de la reputación que alcance en su producto ofertado y las opiniones de la sociedad.

\section{Marco metodológico}

Este estudio se ha centrado en la realización de una revisión bibliográfica de los conceptos más relevantes y significativos que engloban a las cooperativas de enseñanza. Se analizan distintas cuestiones desde la delimitación conceptual como movimiento dentro de la economía social hasta su alternativa educativa y vía emprendedora en tiempos de crisis. Para ello, se ha empleado un estudio de los principales autores destacados en este campo de conocimiento, así como distintos organismos y entidades que prestan servicios en relación con la economía social (Cordero, Manchón y Simancas, 2014).

La metodología que se ha empleado para desarrollar tal investigación, se sustenta en el paradigma cualitativo, basado en el estudio descriptivo (Colás y Buendía, 1998), tanto del análisis de contenido, como método idóneo para estudiar y analizar la temática de forma objetiva y cuantitativa. Así como el uso de una metodología comparada, para establecer una curación o tratamiento de los diferentes textos empleados para la elaboración de nuevos contenidos de rigor científico (Cohen y Manion, 1990).

El principal eje metodológico se centra en la utilización de una metodología descriptiva, la cual ha permitido descubrir y describir las principales teorías del mundo cooperativo a nivel educativo (Bisquerra, 1996).

Un aspecto esencial en el empleo de esta metodología es la reducción del sesgo de subjetividad, para ello se ha llevado a cabo una subjetividad disciplinada (López, 2002). Se han utilizado métodos de autoconciencia, examen riguroso, análisis 
y reflexiones de los contenidos seleccionados tras una profunda y extensa búsqueda bibliográfica, como principales fortalezas alusivas a la validez metodológica.

El marco principal de la investigación se ha ceñido en escritos de autores reconocidos y considerados como expertos en la materia (Sarceda, Santos y Sanjuán, 2017). Se ha hecho un estudio comparativo de documentos de una sola fuente de información en situaciones diversas y documentos de dos o más fuentes mediante una metodología comparada (López, 2002).

Concretamente, la investigación ha abarcado tres fases. Una primera fase en la que se llevó a cabo un método de corte extensivo, caracterizado por el análisis de una gran abundancia de textos de rigor científico. La segunda fase utilizó un método intensivo, en el que se estudiaron pausadamente los aspectos más destacados por los expertos en el mundo cooperativo (Bardin, 1986).

Finalmente, en la tercera y última fase, se utilizó un método cuantitativo, seleccionando las unidades conceptuales más significativas dentro de los diversos documentos analizados (Krippendorf, 1997).

La principal limitación encontrada a nivel metodológico es el avance de la literatura científica, pues la investigación es un campo abierto en continua expansión, lo que dificulta el reporte de todas las fuentes bibliográficas y, por consiguiente, poder abarcar todos los estudios publicados al respecto.

\section{Objetivos de la investigación}

Esta investigación se plantea con el objetivo general de conocer la evolución del cooperativismo en el ámbito educativo, desde sus orígenes hasta la actualidad, a partir de un análisis de la bibliografía existente.

De este derivan los siguientes objetivos específicos:

- Identificar el concepto de cooperativa y cooperativa de enseñanza.

- Establecer las principales metas de las cooperativas de enseñanza.

- Concretar los diferentes tipos de cooperativas de enseñanza.

- Conocer los antecedentes históricos quemarcaron el inicio de estemovimiento.

- Identificar a las cooperativas de enseñanza como alternativa educativa.

- Conocer el cooperativismo como salida profesional en el campo de la educación.

- Determinar la adaptación de las cooperativas a los cambios legislativos producidos en el ámbito educativo. 


\section{Resultados}

\section{Las cooperativas como motor regenerador de la sociedad}

Las cooperativas son el ejemplo más destacado dentro del amplio abanico de empresas de la economía social, toman por bandera las metas sociales y culturales (Gallardo y Castilla, 2015). Son auténticas promotoras de la sociedad, caracterizadas por exportar valores, ética y transparencia en sus acciones (Ademar, Carina, Gallo y Hepp, 2016).

Para Labrador, Rivera y Alfonso (2018), las cooperativas son empresas autosostenibles centradas en la eficiencia y eficacia, debido a la utilización de estrategias para garantizar su estabilidad y crecimiento en el sector donde se ubican. Gómez y Gómez (2014) entienden a las cooperativas como organizaciones económica-sociales, sustentadas por la responsabilidad de sus socios.

La empresa cooperativa nace de la iniciativa y de la agrupación de profesionales que pretenden encontrar una vía de acceso laboral, prolifera en tiempos de recesión económica, donde las ayudas públicas y las ofertas de empleo disponible son escasas (Román, 2014).

Las cooperativas son consideradas organizaciones cuya raíz se centra en la participación de las diferentes personas que las conforman, cuyos nexos establecidos entre ellos fomentan la consolidación de este tipo de entidades en la sociedad, gracias al compromiso, esfuerzo y dedicación del estamento de recursos humanos, dando lugar a uno de los puntos fuertes del sistema cooperativo (Coque, 2008).

Siguiendo los estudios de Coraggio, Acosta y Martínez (2011), Marcuello y Nachar (2013), las cooperativas generan un efecto positivo en la ciudadanía, colocando a las personas en el epicentro de la entidad. Su principal objetivo es el bienestar de éstas por encima del capital y las actividades económicas lucrativas, puesto que estas organizaciones son originadas sin ánimo de lucro.

Según Puentes y Velasco (2009), las cooperativas son las responsables de la regeneración del tejido económico y del desarrollo local de las regiones donde se implantan, fundamentadas en la solidaridad y compromiso de sus miembros.

Siguiendo los principios de la economía social, las cooperativas son asociaciones en las que prima la igualdad de cada uno de sus integrantes. Todos son tomados en consideración para la toma de decisiones que afecten a la entidad, todos los socios tienen el mismo derecho a voz y voto, fomenta el sentido democrático del cooperativismo (Fernández, 2006). Para Pertile (2017), las cooperativas son entidades 
autónomas, porque no dependen de los poderes públicos y de propiedad conjunta, es decir, cada socio es tomado en consideración como dueño activo, responsable y democrático de la organización.

Las cooperativas, en base a los estudios de Martínez-Carrasco y Eid (2017), presentan una gran preocupación por la sociedad y la ciudadanía, intentan generar puestos de trabajo con buenas condiciones laborales; y alcanzan por ello una fama y valoraciones positivas al respecto.

Para Sanchis, Campos y Mohedano (2015), las cooperativas cobran un papel relevante en situaciones donde predomina la escasez de recursos económicos. Son un tipo de empresa muy resistente al mantenimiento de puestos de trabajo, esto se demuestra en el número de entidades, el cual crece en tiempos económicamente difíciles, como se aprecia en la siguiente figura.

\section{Trimestres}

\section{$330 \mathrm{k}$}

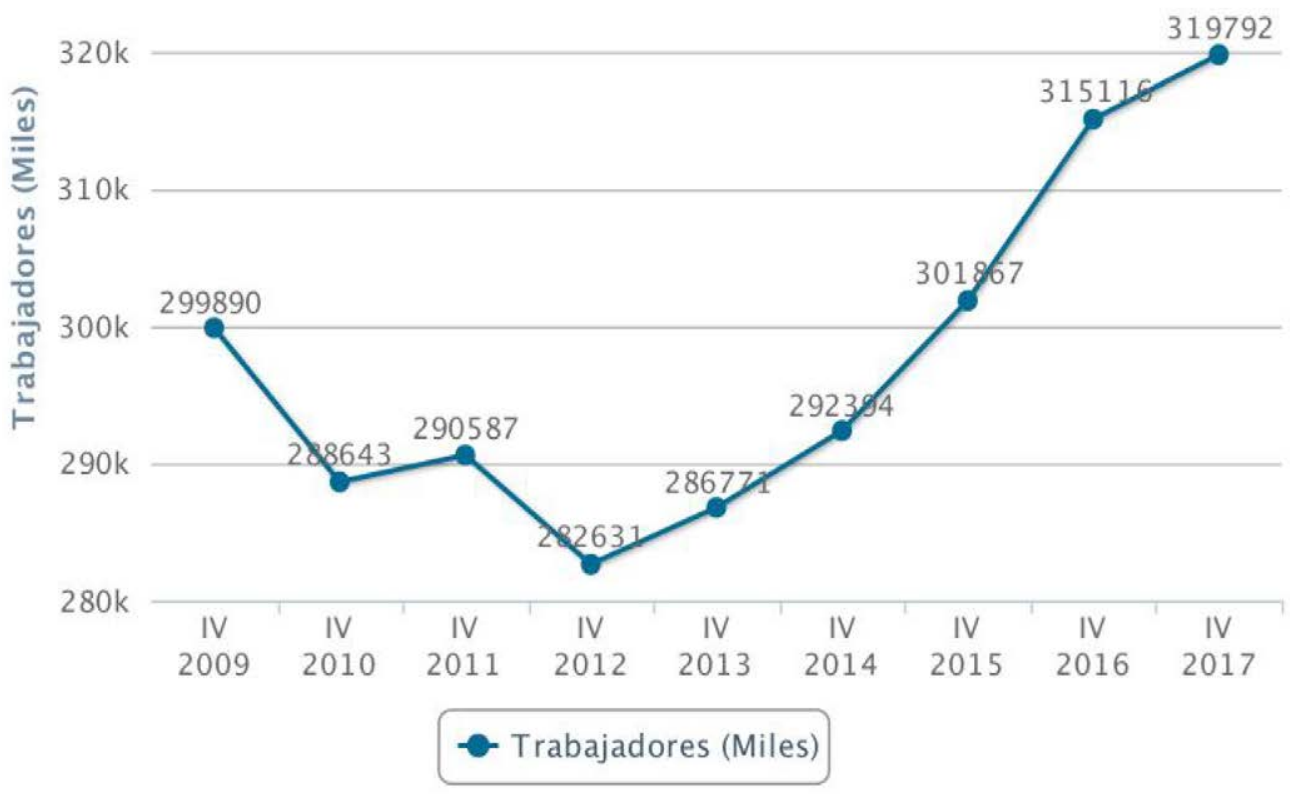

Figura 1. Evolución del número de cooperativas desde el año 2009.

Fuente: Confederación Empresarial Española de la Economía Social (CEPES, 2017).

En el cooperativismo se debe perseguir el fenómeno de la intercooperación entre empresas del mismo sector, a través de relaciones horizontales, para establecer nexos de unión, hacia la mejora de las condiciones laborales, la satisfacción de necesidades 
y resolución de problemas comunes a las diferentes entidades (Martínez, 2012; Prego, Nova y Robaina, 2017). En el caso de la educación, este hecho de unión resulta muy favorecedor para lograr la estabilidad y la expansión como institución educativa.

\section{Las cooperativas de enseñanza}

Como punto de partida, y para una mejor clarificación conceptual, se van a presentar una serie definiciones dadas, a lo largo de la historia, por diferentes leyes y autores que se han interesado en el estudio de este tipo de organizaciones sociales. Fruto de una revisión bibliográfica, se establecen las diversas concepciones sobre el concepto de cooperativa de enseñanza.

Según el artículo 145 de la Ley General de Cooperativas de 1987, en su artículo 145, se define a la cooperativa de enseñanza como "aquellas que desarrollan actividades docentes, en sus distintos niveles y modalidades, en cualquier rama del saber o de la formación técnica, artística, deportiva u otras".

La Ley 27/1999 de Cooperativas, en su artículo 103, define a las cooperativas de enseñanza como "aquellas que desarrollen actividades docentes, en sus distintos niveles y modalidades. Y podrán realizar actividades extraescolares así como prestar servicios que faciliten las actividades docentes".

Se pueden concebir como organizaciones caracterizadas por el compromiso y responsabilidad de sus socios, unidos por un mismo sentimiento de cooperación y superación, para hacer frente a las adversidades de la realidad en la que les han tocado vivir.

Siguiendo a Campos y Navarro (2017), en sus estudios entienden a las cooperativas de enseñanza como un tipo de escuela dentro del sistema educativo que está alcanzando un gran auge, se introducen en el plano de la enseñanza como un "modelo organizacional alternativo". Muchas de ellas actúan como centros concertados, conviviendo entre los centros públicos y privados (Fernández y Muñiz, 2012).

Han experimentado un fuerte desarrollo en las últimas décadas como medida para mejorar la calidad de los centros docentes y de la enseñanza. Atienden siempre a las innovaciones tecnológicas, metodologías, recursos didácticos eficaces, formación permanente de los miembros que componen la estructura social; además de solventar el grave problema de alto índice de paro en este sector.

Son consideradas como medios de difusión de valores entre sus trabajadores y sus "clientes", alumnos y demás personas que componen la comunidad educativa. Esta adquisición de valores redunda en la calidad, prestigio y consideración en la sociedad de este tipo de entidades dentro del colectivo educativo. 
Las cooperativas de enseñanza son una apuesta segura de futuro en el panorama educativo. Son entidades que ofrecen calidad, eficacia en el marco de su proyecto educativo. Persiguen formar a las nuevas generaciones en base a sus valores que las caracterizan. Se presentan en la sociedad como una posible elección dentro de la oferta de centros educativos, siguiendo un modelo laico e integrador de todas las culturas (Inglada, Sastre y Villaroya, 2015).

Trasladan a la gestión del centro educativo herramientas y fórmulas de gestión propias de empresas como una buena planificación, formulación de objetivos, supervisión de la producción y análisis de los resultados obtenidos. Sin estos procesos no se pueden conseguir los fines por los que fueron creadas ni los fines educativos que marcan las leyes.

Se crean bajo un proyecto de empresa caracterizado por su viabilidad y realidad de sus metas. El futuro y vida de la entidad estará condicionado por la adecuación del proyecto a las verdaderas necesidades que haya, tanto en la sociedad como en sus socios. Muchas cooperativas se han visto en la obligación de disolverse ante la falta de realidad mostrada en sus proyectos y cimientos en las que se crearon, al marcarse objetivos intangibles y muy difíciles de conseguir.

Tratan de integrar una homogeneidad entre sus profesionales, no solo a nivel de formación académica sino también de necesidades. Si no se da esta igualdad, el colectivo puede tomar caminos diferentes dentro de la empresa, ocasionando conflictos internos y falta de cooperación, colaboración y trabajo en equipo. En definitiva, la pérdida de valores propios que dificultan tanto la labor docente como la administrativa.

Los socios cooperativistas tienen que convivir con la realización de tareas educativas y empresariales; puesto que son docentes pero al mismo tiempo empresarios de una entidad. Para ello es muy importante seguir formándose, tanto en aspectos relacionados con la docencia como en el ámbito de la gestión y dirección de empresas; que garantice un futuro prometedor y competente (Inglada, Sastre y Villaroya, 2015).

Analizados los estudios realizados por Martínez, Olmedo y Zapata (2013), se concluye que estas sociedades establecen a las personas, sus necesidades y fines en un escalón más elevado de importancia que el capital, por tanto reciben el trato de empresa social, nacidas para proporcionar un servicio a la comunidad que prima con más fuerza que el lucro. Asimismo, han adquirido un peso muy relevante en la comunidad educativa debido a la gran demanda en educación por su obligatoriedad, la escasez de plazas disponibles en la escuela pública y porque su coste es más bajo que en los centros privados.

Para Fernández (2000), las cooperativas de enseñanza gozan de una escasa mortalidad empresarial, a pesar de todas las dificultades que atraviesan en su día a 
día para hacer posible su razón de ser. Deben estudiar las características, fortalezas y amenazas de sus competidores. Aprovechar todas las oportunidades que les brinda el entorno que le rodea para diferenciarse del resto de centros educativos. Estudiar nuevas vías de ingreso, tales como actividades complementarias de carácter lúdico, recreativo y deportivo. Y sobre todo, tener perspectivas de crecimiento empresarial y no estancarse ni conformarse con el rendimiento obtenido, siempre aspirar hacia nuevas metas.

Moreno (2000) considera que las cooperativas de enseñanza, en tanto empresa, tienen que preocuparse, bajo una adecuada gestión y administración, de generar los beneficios necesarios para hacer realidad el proyecto educativo del centro.

En España, la actualidad cooperativa en materia educativa queda reflejada en la siguiente figura.

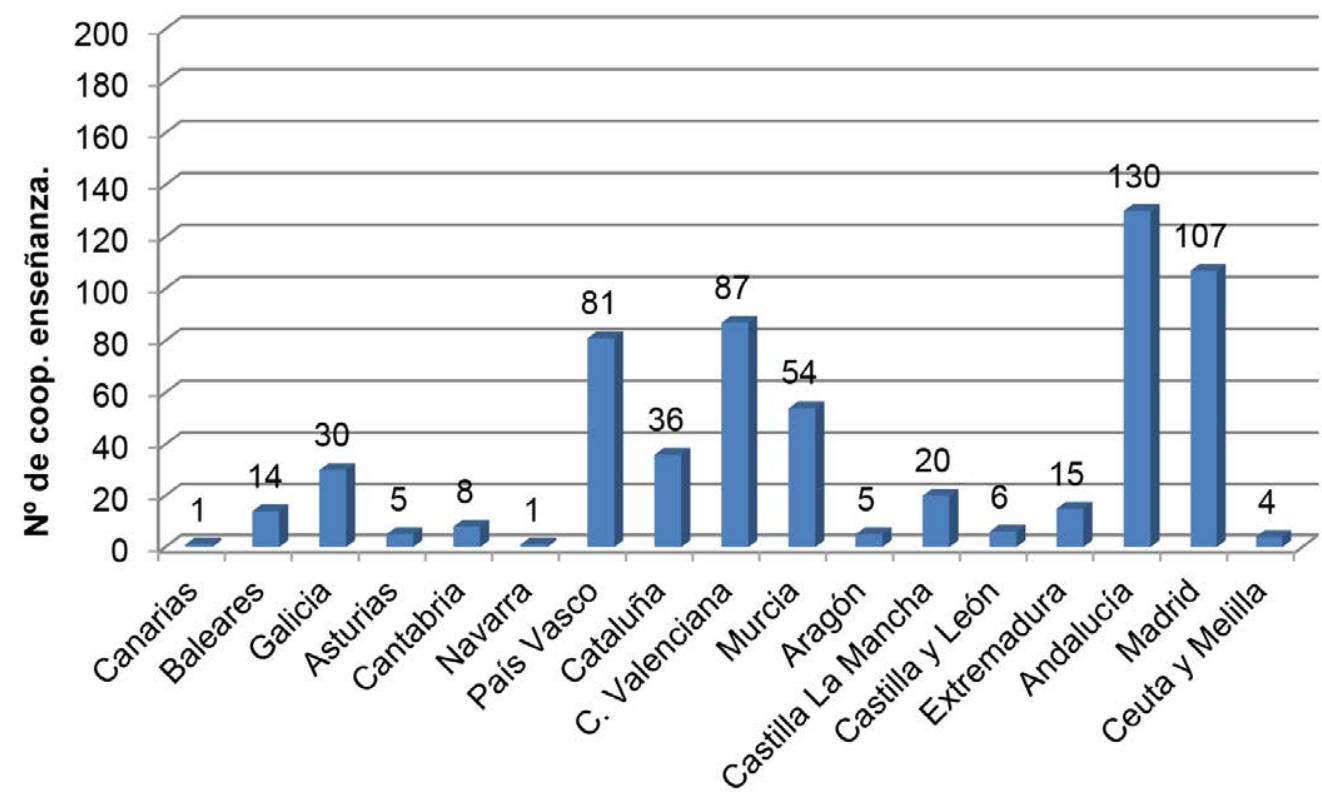

Figura 2. Número de cooperativas de enseñanza en España por autonomías. Fuente: elaboración propia, a partir de datos de UECOE 


\section{Metas de las cooperativas de enseñanza}

Siguiendo los estudios realizados por Delcán (2000), Fernández (2000), Fernández (2000), Fuentes (2004), Moreno (2000), Villalba (2012) e Inglada, Sastre y Villaroya (2015), entre otros, se considera que toda cooperativa de enseñanza ha sido creada para:

- Hacer frente a las necesidades de un grupo de socios-profesores que unidos persiguen alcanzar sus fines.

- Fomentar tanto la creación como el mantenimiento de los puestos de trabajo.

- Gestionar empresarialmente un centro educativo.

- Ofrecer un servicio de calidad a sus usuarios-alumnos.

- Solventar problemas relativos al ámbito educativo como consecuencia de los continuos cambios y avances de la sociedad.

- Desarrollar un proyecto educativo y a la vez empresarial de carácter único y competitivo con el resto de centros que conforman la comunidad educativa de una población.

- $\quad$ Ofrecer una alternativa entre la enseñanza de carácter público y la privada.

\section{Tipología de cooperativas de enseñanza}

Según Fernández (2001) y tomando el rumbo que dicta la Ley 27/1999 General de Cooperativas, se presenta una clasificación de cooperativas de enseñanza. Se establecen tres grandes grupos que son las cooperativas de proveedores, de consumidores y por último, las integrales de educación.

Empezando por el grupo de cooperativas proveedoras de educación, estas aglutinan una única fórmula, que son las de trabajo asociado. Estas entidades son constituidas por un grupo de socios docentes únicamente o desarrollar su existencia en la sociedad con otro tipo de personal no docente, como puede ser el caso de profesionales encargados de la prestación de servicios administrativos a la cooperativa.

El segundo gran grupo de cooperativas de enseñanza lo conforman las consumidoras de educación, que aglutina a las siguientes variantes:

- Cooperativas de padres de alumnos: como bien dice su denominación, estas entidades están compuestas por padres de alumnos, con el fin de mejorar la educación de sus hijos. Se busca una mejor calidad en la 
enseñanza y hacer frente a las debilidades que presenta el centro educativo en el que se encuentran matriculados sus hijos.

- Cooperativas de alumnos mayores de edad: integradas por alumnos que cumplen la mayoría de edad y que persiguen el objetivo de realizar actividades de índole deportiva y cultural en el centro educativo donde se formaron o se siguen formando, si se trata de centros de adultos o formaciones profesionales.

- Cooperativas de alumnos menores de edad: formadas por alumnos de uno o varios centros educativos de una región, cuya meta es la consecución de actividades culturales y deportivas, para el fomento de diferentes valores escasos en la sociedad, como el compañerismo y la empatía, a través de actividades promovidas en los centros de enseñanza.

- Cooperativas de servicios: integradas por diferentes profesionales que se encargan de la prestación de servicios relacionados con la enseñanza, a través de distintas modalidades de formación, como pueden ser los cursos, seminarios, jornadas o clases de refuerzo de alguna materia en concreto.

Por último, el tercer estamento de cooperativas de enseñanza, engloba a las llamadas cooperativas integrales de educación, que reúnen tanto a profesionales de la educación como de cualquier otro ámbito, además de integrar a padres y alumnos. Se convierten en un tipo de cooperativa de naturaleza compleja, en cuanto a su gestión, debido a la convergencia de diversos intereses y fines a perseguir por la entidad.

Todo este entramado cooperativo se da en la sociedad española gracias a la unión de personas comprometidas con la educación y con los valores y fórmulas que ofrece el cooperativismo, para prestar un servicio de calidad a todos los miembros de la comunidad.

\section{Antecedentes históricos de las cooperativas de enseñanza}

Según Fuentes, Lorenzo y Corchón (2002), el cooperativismo, como sistema que aglutina un carácter social y económico, nace en el siglo XIX promovido e impulsado por la revolución industrial. Las primeras cooperativas de enseñanza en España se dieron en el País Vasco y Cataluña, concretamente en la década de los años sesenta, promovidas por padres insatisfechos con el sistema escolar de la época que buscaban alcanzar una educación de calidad mediante el movimiento cooperativo. 
Más adelante, en los años setenta, surgen las cooperativas de trabajo asociado, en las cuales, los propios socios son también los trabajadores del centro escolar. Estas nacen como consecuencia de una crisis económica, en la que algunos docentes de la escuela privada vieron peligrar sus puestos de trabajo y optan por la transformación hacia una cooperativa de enseñanza de trabajo asociado, para poder desempeñar su labor docente, todos unidos por los mismos objetivos, hacer frente a la crisis y mantener sus puestos de trabajo.

El tercer hito histórico se da en la década de los años ochenta, la enseñanza sufre un alto índice de paro por parte del sector del profesorado. Estos toman la decisión de unirse, cooperar, elaborar un proyecto educativo común propio de sus necesidades. Se deciden a fabricar edificios para desempeñar su labor docente. Estamos ante los inicios de la concepción de socio como persona que ha adquirido un puesto de trabajo bajo la fórmula de cooperativa de enseñanza de trabajo asociado.

Para la Unión Española de Cooperativas de enseñanza estos momentos históricos desembocan, en la actualidad, en la creación de numerosas cooperativas de naturaleza educativa. La mayoría de ellas perviven en el presente, como centros educativos competentes e innovadores, aunque, sí es cierto, no todas las cooperativas que se originaron, en la historia de España, han perdurado hasta el presente (UECOE, 2016).

\section{Una alternativa dentro de nuestro sistema educativo}

El sistema educativo español es de naturaleza mixta (Fernández, 2001). En él, coexisten varias modalidades de centros educativos. Estas son, los centros públicos, centros privados y centros concertados, estos últimos pueden ser de naturaleza cooperativa.

Siguiendo a autores como Fernández (1999), Fuentes, Lorenzo y Corchón (2002) las cooperativas de enseñanza surgen como un modelo alternativo en el sistema educativo español, con la intención de mejorar la educación y ofrecer un servicio distinto al resto de centros educativos. Abarcan su ámbito de actuación tanto en la enseñanza reglada como no reglada. Son consideradas entidades democráticas ya que se rigen por estatutos que han sido elaborados por mutuo acuerdo entre todos los miembros de la sociedad cumpliendo con los principios cooperativos. Buscan situarse entre la oferta de la escuela pública y la privada, al ofrecer eficiencia, innovación, crecimiento y calidad del producto final que es la enseñanza (López, Moreno y Fuentes, 2018). 
En la figura 3 se muestran los sectores que refleja la situación actual comparativa entre los distintos tipos de centros de enseñanza. Hay una notoria superioridad de centros públicos, que abarcan un $68 \%$ del total de centros, equivalente a 40295 centros. Seguidos de un $31 \%$ de centros privados que no integran cooperativas, correspondiente a 18617 centros y un escaso 1 \% de cooperativas de enseñanza, referido a un total de 604 centros.



$\square$ Centros privados

- Cooperativas de enseñanza

$\square$ Centros públicos

Figura 3. Total de centros educativos en España en la actualidad. Fuente: elaboración propia, a partir de datos de UECOE

El movimiento cooperativo español en el ámbito educativo es joven, aunque ha asumido cierta importancia en los últimos años como consecuencia de los cambios políticos, sociales y económicos que han acontecido en España (López 2019).

Las consideradas cooperativas de trabajo asociado son las que más se han expandido en cuanto a número de centros en España. Los profesionales de este tipo de centros presentan una doble condición, por un lado, son socios de la cooperativa, unidos por una necesidad común; por otro lado, son los docentes que van a llevar a cabo todo el proceso de enseñanza-aprendizaje con los alumnos (Martínez, 2014).

Habría que aclarar un aspecto sobre este tipo de cooperativas, por un lado, presentan un acercamiento y similitud con la escuela pública en cuanto a ser un servicio público que persigue la igualdad de todos en materia educativa; pero, por otro lado, no deja de ser una titularidad privada que es gestionada por el esfuerzo de un grupo de personas.

Existe un aspecto muy importante que va a marcar el rumbo de la cooperativa y va a hacer que se consigan o no los objetivos marcados y es la actitud de los socios, 
sus ganas, dedicación, vocación, entusiasmo, compromiso con que realicen sus tareas en el día a día (Delcán, 2000; López, Moreno y Fuentes, 2018).

Toda cooperativa necesita una correcta y adecuada coordinación para que todos los miembros trabajen en la misma línea y todos sigan el mismo camino hacia la consecución de la meta propuesta. La cooperación, interacción y el trabajo en equipo de las personas que intervienen en todo este proceso son considerados los pilares fundamentales de este modelo educativo. Si por el contrario, los socios que la componen toman caminos opuestos, esto se verá reflejado en una mayor complejidad para satisfacer las necesidades, un mayor tiempo y esfuerzo, provocando una ausencia de motivación así como el sentimiento de pertenecer a un grupo se verá afectado y, como se ha reflejado anteriormente, la pérdida de la actitud. Todo esto, junto con la falta de cooperación puede suponer el fin de la cooperativa dando lugar a la disolución y liquidación de esta (Fernández, 2003).

SegúnFuentes(2004), paraserconscientes deloquesuponetodoesteentramado, hay que elaborar un proyecto educativo, considerado como la pieza fundamental de la cooperativa en torno a la que girará todo el desarrollo de la educación de los alumnos y que perseguirá una educación para todos, igualitaria, sin discriminación alguna y consiga satisfacer las necesidades de estos. Es muy importante la colaboración y nexo de unión entre la escuela y las familias para fomentar la cooperación y darle importancia a dicho proyecto educativo.

Para Gómez (1975), las cooperativas de enseñanza persiguen una serie de fines para marcarse el camino a seguir. Estos pueden ser materiales, en referencia a las actividades y trabajos que llevan a cabo los alumnos mediante la cooperación. Los fines morales, que persiguen la adquisición de competencias y cualidades como el sentimiento del trabajo bien hecho, la responsabilidad, el orden, respeto hacia los demás y el trabajo en equipo como base de la cooperación. En relación con los fines morales aparecen los psicológicos, gracias al trabajo cooperativo el alumno lleva a cabo una serie de vivencias y situaciones que con las simples tareas escolares no hubieran surgido. Otro de los fines que se buscan son los pedagógicos, además de inculcar una serie de valores para que el alumno se desarrolle completamente como persona, hay que concebir al centro cooperativo como un entorno donde se producen un intercambio de contenidos, aprendizajes para lograr el proceso de enseñanza-aprendizaje. También están los fines prácticos, basados en la idea de intentar que los alumnos se desenvuelvan en su día a día. Los fines sociales se alcanzan gracias a las actividades y eventos socializadores que realizan las cooperativas entre el propio centro y las familias. Además, han de lograr fines económicos de producción, consumo, ejercicios de contabilidad como cualquier otra clase de 
cooperativa, pero por encima de todos estos fines mencionados, se encuentra la finalidad educativa.

Actualmente, las cooperativas de enseñanza se presentan como una de las opciones a elegir dentro de la oferta educativa. Los padres pueden decidir matricular a sus hijos en centros públicos, en privados o declinarse por un centro cooperativo caracterizado por su naturaleza pedagógica-empresarial y sin ánimo de lucro (Fuentes, Lorenzo y Corchón, 2002, López, 2017).

Pedreño y Fernández (1994) consideran que la educación cooperativa es pluralista, es decir, acoge diferentes culturas, defiende la libertad de expresión y es laica. También se caracteriza por ser investigadora y crítica, ya que emplea una metodología basada en la resolución de problemas, apoya las opiniones personales críticas, pero nunca con competitividad. Es no marginadora, atendiendo a las desigualdades y minusvalías. Y es coeducadora, no establece diferencias de sexo, inculca valores como la responsabilidad, la solidaridad, la tolerancia, la paz, el respeto hacia el medio que nos rodea y la cooperación en el proceso educativo. La adquisición de estos valores va a fomentar que el alumno se desarrolle de manera integral y así estar preparado para vivir y trabajar en una sociedad cada vez más compleja.

En lo referente al personal docente, hay que hacer hincapié en la doble naturaleza de los miembros que componen este tipo de cooperativas de trabajo asociado (Monzón, 1995; Arroyo, 2001). Como se ha comentado anteriormente, por un lado, son docentes y, por otro, cumplen con la condición de socio. Por lo tanto, un aspecto esencial en ellos es la formación permanente (Marcelo, 2002); tanto en la vertiente educativa, para mejorar los procesos de enseñanza-aprendizaje mediante el manejo de las nuevas tecnologías de la información y comunicación, como en la económica, para lograr la calidad y eficacia de la gestión y administración de la cooperativa (Delcán, 2000).

\section{Las cooperativas de enseñanza como salida profesional}

La situación laboral española en los últimos años se caracteriza por un descenso en la creación de nuevas empresas, lo que supone una tasa de paro elevada, pero es, a partir de 2015, cuando este panorama cambia y vuelve a resurgir de manera lenta y paulatina la aparición de nuevas entidades (Bel, Fernández, Lejarriaga y Martín, 2016).

La educación en España se concibe como un medio de difícil acceso laboral, en el que muchos son los candidatos que desean optar por cumplir su vocación docente, pero muy pocos lo consiguen y la competencia en formación inicial y complementaria es alta. 
El panorama en el que se encuentra está marcado por un gran número de docentes en situación laboral desfavorecida completamente, ya que no han podido acceder a ningún puesto de trabajo relacionado con la docencia o, simplemente, no han tenido acceso a la escuela pública mediante oposición (Egido, 2010). Este grupo, sumado con la elevada cifra de jóvenes egresados universitarios de las facultades de educación de España, generan una alta cifra de docentes en nuestro país cada año, contribuyendo a que cada vez resulta más complejo desempeñar la labor docente en un centro educativo. Resulta fundamental el fomento entre los más jóvenes de la cultura de creación de nuevas empresas (Bel, Lejarriaga, Fernández y Martín, 2017).

Ambos colectivos presentan una serie de aspectos en común, como pueden ser la aspiración a la escuela pública, un sector que garantiza una larga vida laboral y el desempeño de la docencia, con posibilidad de movilidad entre la oferta de vacantes en los centros del territorio nacional. El gran inconveniente es la escasez de plazas generadas, en contraposición con el gran número de aspirantes que intentan una y otra vez alcanzar un empleo público.

Otro punto de similitud es la desmotivación y el estado de frustración al no encontrar ninguna salida laboral tras años de estudios universitarios, que plantean la idea de buscar otro tipo de empleo, fuera del campo de la educación.

Como alternativa y principal salida laboral de estos profesionales de la educación, se plantea la fórmula del emprendimiento (Melián y Campos, 2010). Es decir, crear su propio centro educativo bajo el amparo de un grupo de personas, unidas por la misma necesidad laboral, que apuestan por el cooperativismo, como herramienta para poner solución a esta difícil situación.

No será un camino fácil, puesto que tendrán que aunar mucho esfuerzo y dedicación para empezar una cooperativa de enseñanza desde sus cimientos, tanto jurídicamente como estructuralmente, en cuanto a instalaciones y equipamientos se requieran.

A continuación se presenta una figura que sirve de síntesis de todo este entramado educacional que viven los profesionales de este sector día a día, tratando de hacer frente a las adversidades para poder desarrollar su tan deseada vocación docente. 


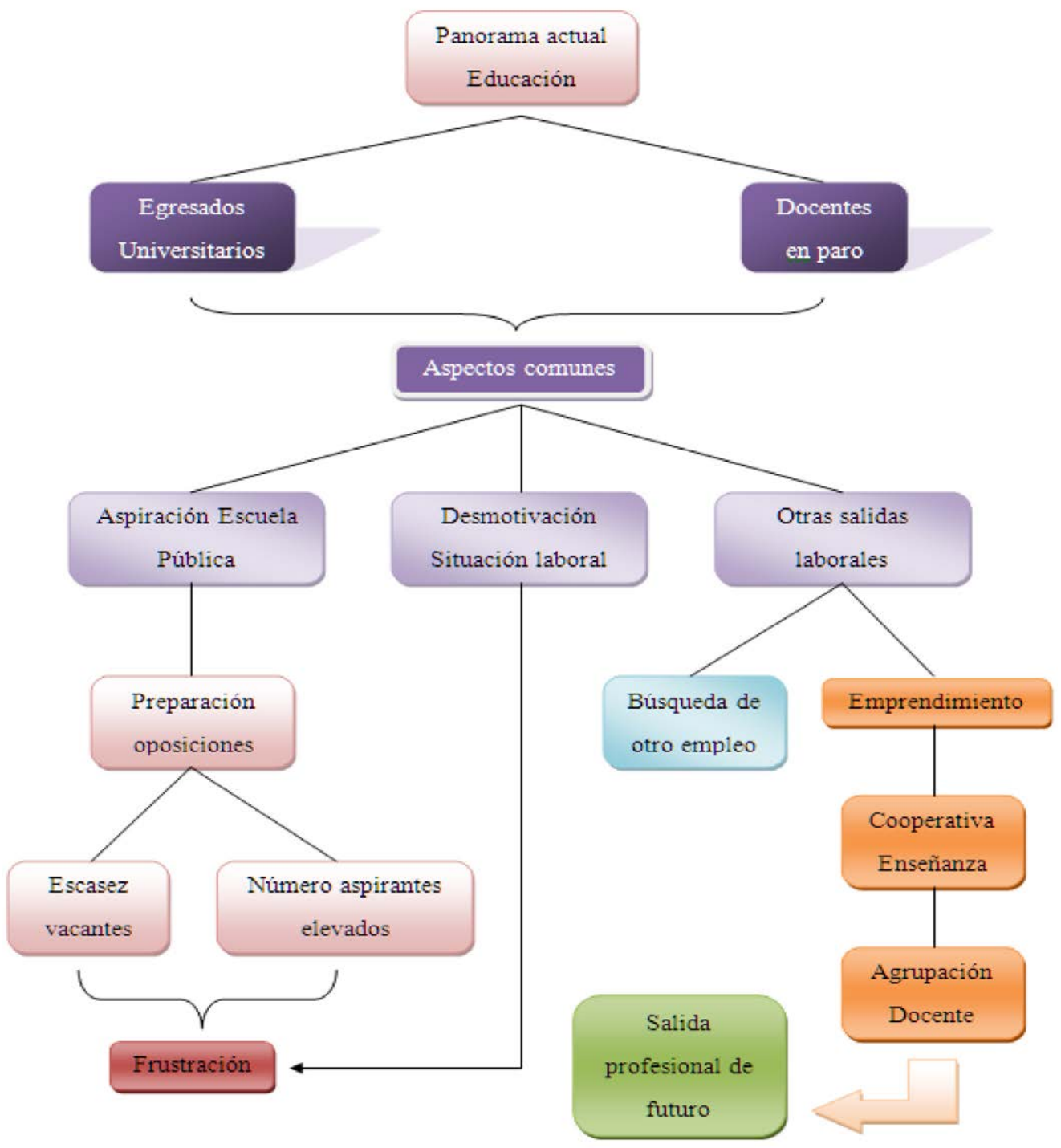

Figura 4. Panorama actual de la educación en España.

Fuente: elaboración propia

\section{Adecuación de la cooperativa a los continuos cambios de las reformas educativas}

El sistema educativo español, debido a los continuos cambios legislativos que se han ido produciendo en las últimas décadas (Berengueras y Vera, 2015), se ha visto perjudicado por la dificultad que supone la adaptación de los centros cooperativos a cada reforma legislativa. Estos cambios ocasionan un gran problema de inestabilidad en la 
enseñanza. Los expertos consideran que para que el sistema educativo progrese, es necesaria la implantación de una ley que perdure en el tiempo y que no sea modificada cada vez que se produce un cambio en el poder ejecutivo del país, ya que, para mejorar, es preciso la estabilidad del sistema (De Puelles, 2008).

Desde la promulgación en 1970 de la Ley General de Educación (L.G.E.) hasta 2013 que se implantó la Ley Orgánica para la Mejora de la Calidad Educativa (L.O.M.C.E.), se han desarrollado siete leyes educativas, cada una con sus peculiaridades y exigencias, que han provocado la constante adecuación de los centros para cumplir legalmente con todos los preceptos formulados en ellas.

Cuando se habla de adecuación, se hace referencia a los cambios en la infraestructura, en los equipamientos y en la contratación de personal especialista en distintos campos para cubrir las necesidades del centro.

Con respecto a la infraestructura de las cooperativas de enseñanza, lo más destacable son las reformas realizadas por estas entidades para la apertura de nuevas instalaciones, aulas, departamentos que han ido exigiendo cada nueva ley, además de las mejoras realizadas en las instalaciones ya existentes que cada cierto periodo de tiempo necesitan ser reformadas para su óptimo mantenimiento.

En cuanto a los equipamientos, se refiere a todo aquel material que se ha tenido que adquirir para ofrecer un servicio de calidad acorde a los cambios legislativos. Como el mobiliario para equipar las nuevas aulas y despachos, instalaciones eléctricas para hacer extensible el uso de las nuevas tecnologías en todo el edificio, como una red tanto cableada como inalámbrica para disponer de una conexión a Internet en todos los dispositivos electrónicos del centro. La inmersión de las tecnologías de la información y comunicación en la enseñanza, junto con la implantación de la escuela 2.0 en los centros educativos, supuso una fuerte inversión de las cooperativas de enseñanza en la compra de dispositivos electrónicos para dotar las aulas con pizarras digitales interactivas, ordenadores portátiles que requieren dichas pizarras, tablets para la gestión docente, plataformas digitales específicas para el tratamiento de datos de carácter educativo. Es decir, una serie de elementos que innovan y aumentan la calidad de los centros de enseñanza.

Otra adecuación es la contratación de personal específico para el tratamiento a la diversidad del alumnado, que se encuentra matriculado en los centros educativos, y así, poder dar respuesta a todo tipo de necesidades y dificultades en el aprendizaje, que están presentes en la escuela.

Todos estos cambios e inversiones realizadas han supuesto un gran problema para este tipo de entidades, debido a la falta de financiación estatal (García, Almeida, Martín y Varela, 2012), que a diferencia de los centros públicos, tienen las cooperativas 
de enseñanza, teniendo que hacer frente, con sus propios medios, a la emisión de pagos de todas las reformas y bienes adquiridos para su correcta solvencia económica.

\section{Conclusiones}

Llegados a este punto, se pretende hacer una recapitulación de las principales ideas que se han ido extrayendo a lo largo de toda la revisión bibliográfica, sirviendo, a modo de síntesis general, de referencia teórica para todos los lectores interesados en el conocimiento de las ideas y concepciones más recientes sobre el mundo cooperativo en las organizaciones de ámbito educativo.

Comenzando por su crecimiento y evolución, hay que resaltar que, en la última década, este movimiento ha originado una media de 600 cooperativas de enseñanza, siendo las Comunidades Autónomas las que más han prosperado en este movimiento, Andalucía, Madrid, Comunidad Valenciana y País Vasco.

El cooperativismo en la educación se considera un movimiento aún joven en España, por su escaso número de cooperativas de enseñanza, frente a la abundancia de centros de carácter público. Uno de los principales motivos de su lenta expansión es la gran inversión económica que supone la creación de una nueva cooperativa, tanto en infraestructura, como en equipamientos y dotación de recursos para poder convertirlo en un centro educativo, de acorde a los preceptos legislativos.

Las personas que deciden emprender e invertir en el mundo cooperativo destacan por su necesidad laboral y escasez de recursos económicos. Por tanto, se afirma que, conforme pasan los años, se va aumentando el número de cooperativas, por necesidad de sus miembros, pero de manera muy lenta por la escasez de recursos.

El movimiento cooperativo está basado en la concepción de equipo, integrado por personas unidas por una misma necesidad que pretenden, mediante su esfuerzo, dedicación, colaboración y humildad, buscar una solución a la realidad que están viviendo, siendo la causa que más propicia este movimiento la creación de puestos de trabajo, para poder subsistir en este mundo tan competitivo y afectado por una profunda crisis económica.

Esta clase de centros educativos pretenden situarse entre las opciones educativas presentes en la sociedad. Se encuentran ocupando un tercer puesto por su número de centros a nivel nacional, detrás de los centros públicos y privados. Quieren mostrarse como una alternativa a la bipolaridad educativa, que antiguamente se daba en España.

Por último, se recomienda fomentar, en las distintas Facultades de Educación, los contenidos referentes al mundo cooperativo, para que los estudiantes y futuros 
docentes conozcan las características de este tipo de entidades, así como sus fórmulas de emprendimiento, a través de la economía social, como vía para alcanzar un puesto de trabajo.

\section{Referencias}

Ademar, H., Carina, S., Gallo, G. y Hepp, M. (2016). Educación, Cooperativismo y Cooperativas Escolares en la provincia de Córdoba Argentina (2006-2015) y su vinculación con el movimiento cooperativo. Sociales Investiga, 2(2), 22-36.

Arroyo, P. (2001). Cooperativas de trabajo asociado, alternativas de empleo. Revista de Estudios de Juventud, 51, 47-55.

Bardin, L. (1986). El análisis de contenido. Madrid: Akal.

Bel, P., Fernández, J., Lejarriaga, G. y Martín, S. (2016). La iniciativa emprendedora como base para la creación de empresas de participación. Un instrumento para la innovación social. Cooperativismo \& Desarrollo, 108, 123-141. doi: 10.16925/co.v24i108.1267

Bel, P., Lejarriaga, G., Fernández, J. y Martín, S. (2017). La necesaria coordinación entre los diferentes niveles educativos para el estímulo de la iniciativa emprendedora: un estudio basado en las cooperativas de enseñanza españolas. En A. Daniel. Conferência sobre Educação para o Empreendedorismo. Aveiro: Universidad de Aveiro.

Berengueras, M. y Vera, J. (2015). Las Leyes de educación en España en los últimos doscientos años. Supervisión 21, revista de educación e inspección, 38, 1-23. Recuperado de: http://usie. es/las-leyes-de-educacion-en-espana-en-los-ultimos-doscientos-anos/

Bisquerra, R. (1989). Método de investigación educativa. Guía práctica. Barcelona: Ediciones CEAC.

Campos, V. y Navarro, C. (2017). Participación en redes organizacionales y uso de las tecnologías de la información y la comunicación, un estudio de su impacto en los resultados de las cooperativas de enseñanza de la comunidad valenciana. REVESCO. Revista de Estudios Cooperativos, 124, 32-46.

Cohen, L. y Manion, L. (1990). Métodos de investigación educativa. Madrid: La muralla.

Colás, B. y Buendía, L. (1998). Investigación Educativa. Sevilla: Ediciones Alfar. 
Confederación Empresarial Española de la Economía Social (CEPES). (2017). Estadísticas estatales. Recuperado de: https://www.cepes.es/social/estadisticas\&t=cooperativas

Coque, J. (2008). Puntos fuertesy débiles de las cooperativas des de un concepto amplio de gobierno empresarial. REVESCO. Revista de Estudios Cooperativos, 95, 65-93.

Coraggio, J., Acosta, A. y Martínez, E. (2011). Economía social y solidaria: El trabajo antes que el capital. Quito, Ecuador: Ediciones Abya-Yala.

Cordero, J. M., Manchón, C. y Simancas, R. (2014). Retention and its condicioning factors in Spain. Revista de Educación, 365, 12-37. doi: 10.4438/1988-592X-RE-2014-365-263.

De Puelles, M. (2008). Las grandes leyes educativas de los últimos doscientos años. Participación educativa, 7, 7-15. Recuperado de: https://sede.educacion.gob.es/publiventa/d/14200/19/0

Delcán, B. (2000). Reflexiones sobre las claves de gestión futuras de las Cooperativas de enseñanza: calidad, mejora continua y servicio como base de la excelencia educativa. REVESCO, Revista de Estudios Cooperativos, 71, 149-170.

Egido, I. (2010). El acceso a la profesión docente en España en perspectiva europea. Algunas reflexiones orientadas a la mejora de la selección del profesorado. Educación XX1, 13, 47-67.

Fernández, F. (2000). Realidad actual y perspectivas de las cooperativas de enseñanza. REVESCO, Revista de Estudios Cooperativos, 71, 77-82.

Fernández, J. (1999). Las sociedades cooperativas en la educación ante la nueva concepción del Estado en la provisión de servicios educativos. REVESCO, Revista de Estudios Cooperativos, 67, 71-88.

Fernández, J. (2001). Estudio de los flujos económico-financieros de las sociedades cooperativas en la educación: un enfoque de concentración empresarial. Madrid, España: Universidad Complutense de Madrid.

Fernández, J. (2001). La cantidad y calidad del empleo generado por las sociedades cooperativas que prestan servicios educativos de la Comunidad Autónoma de Madrid. Cuadernos de estudios empresariales, 11, 161-186.

Fernández, J. (2003). La participación, factor determinante de la calidad educativa de los centros docentes cooperativos. REVESCO, Revista de Estudios Cooperativos, 79, 35-58. 
Fernández, M. (2006). Las cooperativas: organizaciones de la economía social e instrumentos de participación ciudadana. Revista de Ciencias Sociales (Ve), 12(2), 237-253.

Fernández, R. y Muñiz, M. (2012). Colegios concertados y selección de escuela en España: un círculo vicioso. Presupuesto y gasto público, 67, 97-118.

Fuentes, A. M. (2004). Las cooperativas de enseñanza:(un estudio de las cooperativas de trabajo asociado). Ceuta: Universidad Nacional de Educación a Distancia, Centro Asociado de Ceuta.

Fuentes, A. M., Lorenzo, M. y Corchón, E. (2002). Las cooperativas de enseñanza como tercera vía dentro de nuestro sistema educativo: las cooperativas de trabajo asociado. Percepciones de sus directivos. Enseñanza, 20, 51-84.

Gallardo, D. y Castilla, F. (2015). Modelo de gestión para la responsabilidad social en cooperativas. Economía industrial, 396, 139-149.

García, E., Almeida, F., Martín, G. y Varela, S. (2012). Asesoramiento contable y de gestión para cooperativas de trabajo. Zamora: COODE, Cooperación para el desarrollo.

Gómez, M. A. (1975). Informe sobre Cooperativas. Revista de la Asociación de Estudios Cooperativos. Madrid.

Gómez, J. y Gómez, J. (2014). Fundamentos para la evaluación de la gestión en empresas cooperativas. Avances, 16(1), 18-27.

Inglada, E., Sastre, J. M. y Villaroya, B. (2015). El cooperativismo en la educación. REVESCO, Revista de Estudios Cooperativos, 118, 122-147. doi: 10.5209/rev_REVE.2015.n118.49059

Krippendorf, K. (1997) Metodología de análisis de contenido. Teoría y Práctica. Barcelona: Paidós.

Labrador, O., Rivera, C. y Alfonso, J. (2018). La eficiencia y eficacia socioeconómica de las cooperativas: enfoque desde la actualización del modelo económico cubano. Avances, 20(1), 11-26.

Ley 27/1999 (1999). Ley de Cooperativas. Madrid, 17 de julio de 1999, núm. 170, pp. 27027-27062.

Ley3/1987. (1987). Ley General de Cooperativas. Madrid, 8deabril de 1987, núm.84, pp. 10452-10487.

López, F. (2002). El análisis de contenido como método de investigación. XXI. Revista de educación, 4, 167-179. Recuperado de: http://rabida.uhu.es/dspace/handle/10272/1912 
López, J. (2017). El fenómeno del liderazgo en los centros de enseñanza de naturaleza cooperativa. En J.M. Alcántara, M. Bermúdez, F.J. Blanco, y J.M. Heredia. (Eds.). Investigación e innovación en el ámbito universitario. Tendencias ante los retos actuales de la sociedad. Madrid: EOS.

López, J. (2019). El fenómeno del liderazgo en los centros educativos. Un estudio de las cooperativas de enseñanza en España. Revista Internacional de Educación y Aprendizaje, 6(3), 165-175.

López, J., Moreno, A. J. y Fuentes, A. (2018). La evolución del movimiento cooperativo desde su origen hasta la actualidad a través de su máximo exponente: La sociedad cooperativa. European Scientific Journal, 14(29), 53-74.

López, J., Moreno, A. J., y Fuentes, A. (2018). Influencia del género y la antigüedad profesional en el liderazgo directivo en las Cooperativas de enseñanza. Revista Innova, 3(10), 1-19.

Marcelo, C. (2002). Los profesores como trabajadores del conocimiento. Educar, 30, 27-56.

Marcuello, C. y Nachar, P. (2013). La sociedad cooperativa: Motivación y coordinación. Un análisis desde las teorías económicas de la empresa y la economía social. REVESCO. Revista de Estudio Cooperativos, 110, 192-222.

Martínez, A. (2012). Sobre el principio de cooperación entre cooperativas en la actualidad. Boletín de la Asociación Internacional de Derecho Cooperativo, 46, 133-146.

Martínez, I. R. (2014). La cooperativa de trabajo asociado de trabajadores y el sentimiento de pertenencia de su profesorado. Qurriculum: Revista de teoría, investigación y práctica educativa, 27, 85-104. Recuperado de: http://publica.webs.ull.es/publicaciones/volumen/ revista-qurriculum-volumen-27-2014/

Martínez, I. M., Olmedo, I. y Zapata, J. (2013). Reputación percibida por el profesorado de las cooperativas de educación: medición e influencia. CIRIEC-España, Revista de Economía Pública, Social y Cooperativa, 77, 237-260. Recuperado de: http://www.ciriec-revistaeconomia.es/ index.php?id_pagina=7\&id_revista=85

Martínez-Carrasco, F. y Eid, M. (2017). El nivel de conocimiento y la reputación social de las empresas cooperativas. El caso de la Región de Murcia. CIRIEC-España. Revista de economía pública, social y cooperativa, 91, 5-29.

Melián, A., y Campos, V. (2010). Emprendedurismo y economía social como mecanismos de inserción sociolaboral en tiempos de crisis. REVESCO, Revista de Estudios Cooperativos, 100, 43- 
67. Recuperado de: http://revistas.ucm.es/index.php/REVE/article/view/REVE1010130043A $/ 18650$

Monzón, J. L. (1995). Las cooperativas de trabajo asociado ante la reforma de los principios cooperativos. REVESCO, Revista de Estudios Cooperativos, 61, 47-52.

Moreno, R. (2000). Realidad actual y perspectivas de futuro para las sociedades cooperativas en la educación. REVESCO, Revista de Estudios Cooperativos, 71, 83-91.

Pedreño, J. A. y Fernández, F. (1994). Las cooperativas de enseñanza en España. Madrid: UECOE.

Pertile, V. (2017). Las cooperativas en el contexto de las organizaciones de la economía social: breve reseña histórica. Características generales. Geográfica digital, 10(20), 1-18.

Prego, J., Nova, A. y Robaina, L. (2017). Formas de integración cooperativa y sus principales técnicas de realización. La intercooperación cooperativa. Estudios del Desarrollo Social: Cuba y América Latina, 5(3), 85-99.

Puentes, R. y Velasco, M. (2009). Importancia de las sociedades cooperativas como medio para contribuir al desarrollo económico, social y medioambiental, de forma sostenible y responsable. REVESCO. Revista de Estudios Cooperativos, 99, 104-129.

Román, C. (2014). Las cooperativas españolas y los ciclos económicos. Un análisis comparado. CIRIEC-España, Revista de Economía Pública, Social y Cooperativa, 80, 76-92.

Sanchis, J. R., Campos, V., y Mohedano, A. (2015). Factores clave en la creación y desarrollo de cooperativas. Estudio empírico aplicado a la Comunidad Valenciana. REVESCO. Revista de Estudios Cooperativos, 119, 183-207.

Sarceda, M.C., Santos, M.C. y Sanjuán, M.M. (2017). La Formación Profesional Básica: ¿alternativa al fracaso escolar? Revista de Educación, 378, 78-102. doi: 10.4438/1988-592X-RE-2017-378-362.

Unión Española de Cooperativas de Enseñanza (UECOE). (2016). ¿Qué es una cooperativa? Recuperado de: https://www.cepes.es/principal/members/102_union-espanola-cooperativas-ensenanza-uecoe

Villalba, A. (2012). Las Cooperativas de Enseñanza: una perspectiva jurídica. Trabajo Final de Grado: Universidad de Valladolid. Facultad de Ciencias del Trabajo. Recuperado de: https://uvadoc. uva.es/bitstream/10324/1821/1/TFG-L73.pdf 